\title{
Detecting and targeting senescent cells using molecularly imprinted nanoparticles
}

Akang E. Ekpenyong-Akiba ${ }^{1 ¥}$, Francesco Canfarotta ${ }^{2 ¥}$, Bashar H. Abd ${ }^{3 \mp}$, Marta Poblocka $a^{1 \uparrow}$, Mireia Casulleras ${ }^{1}$, Laura Castilla-Vallmanya ${ }^{1}$, Gabriella Kocsis-Fodor ${ }^{1}$, Michael E. Kelly ${ }^{4}$, Justyna Janus ${ }^{4}$, Mohammad Althubiti ${ }^{1,5}$, Elena Piletska ${ }^{3}$, Sergey Piletsky ${ }^{3}$ and Salvador $\operatorname{Macip}^{1 *}$

${ }^{1}$ Mechanisms of Cancer and Aging Laboratory, Department of Molecular and Cell Biology, University of Leicester, Leicester, UK. ${ }^{2}$ MIP Diagnostics Ltd., Fielding Johnson Building, University of Leicester, UK. ${ }^{3}$ Department of Chemistry, University of Leicester, Leicester, UK. ${ }^{4}$ Preclinical Imaging Facility, Core Biotechnology Services, College of Life Sciences, University of Leicester, Leicester LE1 9HN. ${ }^{5}$ Department of Biochemistry, Faculty of Medicine, Umm Al-Qura University, Mecca, Saudi Arabia.

¥These authors contributed equally to this paper.

TThese authors contributed equally to this paper.

*Corresponding authors:

Salvador Macip. Department of Molecular and Cell Biology, University of Leicester, University Road, Leicester, LE1 7RH, UK. sm460@leicester.ac.uk, Phone: +44 (0)116 229 7113.

Francesco Canfarotta. MIP Diagnostics Ltd., Fielding Johnson Building, University of Leicester, UK. fc114@leicester.ac.uk

Keywords: Senescence, ageing, molecular imprinting, nanoparticles. 


\section{ABSTRACT}

The accumulation of senescent cells in tissues contributes to pathophysiological conditions such as fibrosis, diabetes, cancer, Alzheimer's and ageing. Consistent with this, clearance of senescent cells prolongs lifespan and healthspan in animals. Detecting senescent cells in humans could therefore have a significant diagnostic and prognostic impact. However, identifying senescent cells has proven to be complex. To address this issue, we characterized and validated a panel of novel membrane markers of senescence. Here, we show the application of molecularly imprinted nanoparticles (nanoMIPs) against an extracellular epitope of one of these markers, B2M, to detect senescent cells in vitro and in vivo. We show that nanoMIPs do not elicit toxic responses in the cells or in mice and successfully differentiate young from old animals. Importantly, nanoMIPs loaded with drugs can specifically kill senescent cells. Our results provide a proof-of-principle assessment of novel approaches for senescent cell detection and clearance with potential clinical relevance. 
Senescence, a complex cellular program in which cells enter a state of permanent cell cycle arrest while remaining metabolically active, has important roles in tumour suppression ${ }^{1}$. It is established as a result of different events, including telomere shortening after a number of cell divisions (replicative senescence) or the response to damage (stress-induced premature senescence $)^{2,3}$. Expression of oncogenes can also trigger it (oncogene-induced senescence), underscoring the role of senescence as a defence against neoplastic transformation ${ }^{1}$. Indeed, senescent cells in vivo are often observed in pre-malignant stages of tumours, after which they disappear, which suggests that the senescent barrier needs to be overcome in order to progress into full malignancy ${ }^{4-6}$.

Senescence has been associated with age-dependent organismal changes, and the progressive increase of senescent cells with time is known to contribute to the functional impairment of different organs typically seen in ageing ${ }^{7}$. More recently, it has been shown that it also plays an important role in fibrosis ${ }^{8}$ and, paradoxically, tumour progression ${ }^{9}$, and that it may be involved in cataracts, obesity, diabetes, Alzheimer's and Parkinson’s diseases, arthritis, atherosclerosis and other conditions ${ }^{10}{ }^{11}$. This supports the hypothesis that senescence is an antagonistically pleiotropic process, with beneficial effects in the early decades of life of the organism (in development ${ }^{12}$, tissue repair ${ }^{13}$ and as a tumour suppressor mechanism) but detrimental to fitness and survival in later stages, at least in part due to the paracrine promotion of a chronic inflammatory state ${ }^{14}$.

Since the accumulation of senescent cells is involved in many pathologies, quantifying the number of these cells present in a tissue could have diagnostic and prognostic benefits, and provide the basis for therapeutic clearance strategies. Despite a number of available options, none of the current markers of senescence are sufficiently specific ${ }^{3,15} 16$. Although increased expression of intracellular and/or secreted proteins has often been used as a surrogate marker of senescent cells ${ }^{17}$, their most distinctive measurable feature is a particular enzymatic activity, 
known as senescence associated- $\beta$-galactosidase $(\mathrm{SA}-\beta-\mathrm{Gal})^{18}$. This is still the standard for senescence detection, despite the fact that high cell confluence and certain types of stress can also induce it, leading to potential false positives ${ }^{19}$. Using mass spectrometry on well-studied cellular models ${ }^{20}$, we identified a number of proteins highly expressed in the plasma membranes of senescent cells, which are part of the still poorly characterized senescent surfaceome $^{21}$. We validated several that showed increased senescence-related expression in vivo and in vitro and had extracellular epitopes ${ }^{21}$, and we proposed to use them as tools to detect senescent cells ${ }^{22}$.

A novel technology that could be used for this purpose are the molecularly imprinted nanoparticles (nanoMIPs), nanostructured polymeric particles with one binding site for a target molecule ${ }^{23}$. The solid-phase approach employed in the production of nanoMIPs relies on the covalent immobilisation of the template molecule on a solid support (e.g. glass beads of an average diameter of 75-90 $\mu \mathrm{m})$. This support bearing the immobilized template is placed in contact with the monomer mixture, and polymerisation is initiated under conditions that promote the formation of polymer nanoparticles. After polymerisation, the solid support acts as an affinity medium to isolate the high-affinity nanoMIPs from the remaining monomers, oligomers and low-affinity polymers, which are removed by washing the beads under conditions in which those nanoparticles with high affinity remain attached. Because of this purification step, nanoMIPs possess high affinity and specificity toward their targets and exhibit a homogeneous distribution of binding site affinities ${ }^{24}$.

Here, we used nanoMIPs, targeted against one of the senescence membrane markers we previously described, B2M, to identify and clear senescent cells. We show that they are not toxic and propose that nanoMIPs have the potential to be used as diagnostic, prognostic and therapeutic tools in pathologies characterized by the accumulation of senescent cells. 


\section{RESULTS}

Generation of nanoMIPs against makers of senescence. We hypothesized that nanoMIP technology could provide a new approach to specifically detect senescent cells. In order to test this idea, we used a solid-phase approach based on epitope imprinting to target an epitope of $\beta_{2}$ microglobulin (B2M), an extracellular component of the MHC class I molecules ${ }^{25}$ and a newly identified marker of senescence ${ }^{21}$. The chosen epitope, aminoacids 101-115 (Figure 1A and Supplementary Figure 1A), was fixed on the surface of microsized glass beads via thiol coupling, to allow oriented immobilisation of the peptides, as previously described ${ }^{26}$. This was achieved by adding a cysteine at the C-terminus of the peptide and using succynimidyl-iodo acetate as linker between the peptide and the surface-modified glass beads. To produce nanoMIPs, we utilized a mixture of monomers previously optimized specifically to imprint peptides and proteins in mild aqueous conditions ${ }^{24}$. The generated B2M nanoMIPs had an average hydrodynamic diameter in deionized water of $313 \mathrm{~nm}$, as measured by dynamic light scattering, and transmission electron microscopy showed an average size of $133 \mathrm{~nm}$ (Figure 1B).

B2M-targeted nanoMIPs recognize senescent cells in culture. We next tested the B2M nanoMIPs in an inducible model of senescence, the EJ bladder cancer cells with a tetracycline(tet)-regulatable p16 expression systems (EJp16). These cells undergo senescence after gene induction by tet removal ${ }^{20}$ and are representative of activation of the pRb-p16 axis, one of the main pathways involved in senescence ${ }^{27}$. Moreover, clearing p16-expressing senescent cells has been shown to ameliorate age-related pathologies ${ }^{28-30}$, which underscores the relevance of our model. As expected, B2M was upregulated when cells became senescent (Figure 2A). Incubation of control and senescent cells with fluorescein-tagged B2M nanoMIPs 
showed a selective detection of senescent cells, as measured by FACS (Figure 2B). Fluorescent microscopy confirmed that the nanoMIPs preferentially accumulate in senescent cells (Figure 2C) and that they specifically bind to their membrane (Figure 2D). These results show that nanoMIPs imprinted for a membrane marker can selectively recognize senescent cells in culture.

Targeted clearance of senescence cells using B2M nanoMIPs. We next investigated whether the B2M nanoMIPs could also be used to specifically clear senescent cells through targeted delivery of toxic compounds, which could be a senolytic approach with reduced off-target effects. First, we tested whether the nanoMIPs had any cytotoxic effect on their own. As shown in Figures 3A-B, long term exposure to the B2M targeted nanoMIPs did not affect the viability and survival of either control or senescent cells, confirming that the nanoparticles were not toxic under these conditions. This is consistent with what has previously been demonstrated for other nanoMIPs ${ }^{31}$. We also observed that the nanoMIPs internalized after binding to B2M on the surface of senescent cells and accumulated in the cytosol, often forming perinuclear aggregates (Figure 3C). This supported the hypothesis that nanoMIPs could be used to deliver drugs into cells. To further test this, we generated B2M nanoMIPs loaded with dasatinib, a senolytic drug ${ }^{32}$. We observed that senescent EJp16 cells can be killed by dasatinib and other senolytic drugs, but only at high concentrations, while most of them had no effect on control cells (Figure 3D). Thus, we postulated that a targeted delivery using nanoMIPs could increase the sensitivity of the cells to the drug, while minimizing the impact to other cells. As shown in Figure 3E, dasatinib-containing nanoMIPs were able to importantly reduce the viability of senescent EJp16 cells, while the proliferating controls were significantly less affected. These proof-of-principle experiments demonstrate that nanoMIPs can selectively kill senescent cells by delivering a cytotoxic drug into them. 
In vivo detection of senescent cells by B2M nanoMIPs. We finally studied whether nanoMIPs can also recognize senescent cells in vivo. We observed that the administration of B2M targeted nanoMIPs did not significantly affect the weight of the animals when given orally, intraperitoneally or intravenously during a 14 day follow-up after treatment, suggesting that they had no important effects on their general health (Figure 4A). We also found that the liver and kidney functions were preserved (Figure 4B). Moreover, these mice were assessed daily to measure any distress symptoms and no changes were reported during the 14-day period (Supplementary Table 1). This supports previous reports that suggested that nanoMIPs were safe to be delivered intravenously ${ }^{33}$.

Since the B2M epitope used to generate the B2M MIPs is well conserved between human and mouse (Supplementary Figure 1B) we utilized the same B2M nanoMIPs described above for the in vivo tests. As shown in Figure 5A, nanoMIPs tagged with DyLight 800 NHS Ester and injected intravenously into C57/BL6J mice did not show any fluorescence in young mice ( 2 month old) 2 hours after the treatment, similar to what was observed in the untreated control. On the other hand, old mice (11 month) were positive for a fluorescent signal, suggesting that the nanoMIPs were bound to senescent cells and thus were not cleared from the animals. The accumulation of the nanoMIPs was preferentially in the intestine, specifically the jejunum (Figure 5B and Supplementary Figure 2C). This pattern is consistent with an increased presence of senescent cells in the gastrointestinal tract of old mice ${ }^{34}$. When mice were imaged post-mortem after opening the abdominal cavity, old mice showed a diffuse pattern of fluorescence 4-fold higher than the background levels of young and untreated mice (Figure 5C). Similar results were obtained with nanoMIPs tagged with Alexa Fluor 647, although the shorter wavelength used to image this nanoMIPs led to a higher background fluorescence in control mice (Supplementary Figure 2A-C). Our results suggest that B2M 
nanoMIPs accumulate in senescent cells in vivo, thus acting as a marker for aged tissues that could have clinical relevance.

\section{DISCUSSION}

In recent years, the accumulation of senescent cells has been shown to play a significant role in triggering or maintaining several pathologies ${ }^{10}$. This provides a rationale for devising strategies to detect the presence of senescent cells in tissues, which could be used for diagnostic and prognostic purposes. A key limiting factor for this is the ability to identify senescent cells, although novel techniques are being developed to quantitate their presence ${ }^{35}$. We hypothesized that (i) senescent cells would have a particular pattern of expression of cell surface proteins (the senescent surfacesome) and that (ii) this could be used to recognise these cells both in vitro and in vivo. We characterized several extracellular epitopes preferentially present in senescent cells $^{21}$ that could be used to detect them ${ }^{22}$. In the current study, we tested the potential clinical relevance of our findings by using a new strategy that takes advantage of the senescent surfacesome to locate and eliminate these cells.

We developed a nanoparticle that recognizes B2M, one of the proteins we previously identified as a membrane marker of senescence ${ }^{21}$, using nanoMIP technology. These "plastic antibodies” represent an entirely new compound class, the production of which does not involve the use of animals and it is more cost-effective compared to actual antibodies. They can also withstand harsher conditions and have a longer shelf life. NanoMIPs are currently applied as recognition elements in both optical and electrochemical sensors and assays ${ }^{36} 37$, for instance as replacement of antibodies in ELISA-like assays ${ }^{39} 4041$. Also, their production can be easily automated to improve batch-to-batch consistency ${ }^{23}$. NanoMIPs have already been 
successfully employed as imaging tools in vivo, thus demonstrating their safety and potential diagnostic use ${ }^{42}$.

The B2M nanoMIPs showed a selective binding to senescent cells, proportional to the amount of B2M protein expressed on their surface. On their own, the nanoMIPs had no shortterm toxic effects, a feature that could be exploited to use the nanoparticles as drug delivery mechanisms. Our data shows that the specificity of nanoMIPs allows them to carry a toxic payload into senescence cells with a reduced effect in normal cells. Although this difference is ample and significant, the therapeutic window would have to be further increased before testing these tools in vivo with minimal side effects. These proof-of-principle results could be the basis for designing improved and more specific nanoMIP-drug combinations that could eventually be used in mouse models.

Our experiments also show that fluorescently-tagged nanoMIPs could be used to detect senescent cells in vivo and that, importantly, are not toxic when injected at single doses. Although B2M has basal levels of expression in several organs, we found that it can be successfully used to differentiate young from old mice. The B2M nanoMIPs preferentially accumulated in the abdominal cavity of old mice, which could indicate a higher presence of B2M-expressing senescent cells in the GI tract, as well as liver and spleen. Further experiments will be needed to better understand the localization of these cells in the body. Also, the choice of the right fluorophore needs to be considered carefully. Our results show that a wavelength of $647 \mathrm{~nm}$ is not as useful as $800 \mathrm{~nm}$ because much of the emitted light at low wavelength is absorbed by tissue (specifically haemoglobin). This reduces the sensitivity of the imaging technique and makes it more difficult to separate true fluorophore signal from background tissue signal using post-processing methods (spectral unmixing).

It would also be important to design nanoMIPs against other members of the senescent surfacesome and characterize their accumulation in vivo, not only in ageing animals but also in 
mouse models of senescent-related diseases. We have previously shown that the expression of cell surface senescent markers is variable depending on the tissue of origin and the mechanism of induction of senescence ${ }^{21}$. Therefore, it is expected that not all senescent cells would display a significant increase in B2M expression. This information will have to be taken into account when designing and using nanoMIPs for detection of senescence in specific situations. It would be necessary to determine which of the known membrane markers of senescence is more expressed in each condition of interest, as well as consider the off-target expression to reduce potential false negatives. This could provide an array of specific probes to be used as diagnostic, prognostic and therapeutic tools. Of note, mesoporous silica nanoparticles (MSN) capped with a galacto-oligosaccharide have also been used to deliver cytotoxic drugs to senescent cells, in this case using $\beta$-galactosidase as a target ${ }^{43}$. Intracellular epitopes could be an alternative to our approach, although the fact that $\beta$-galactosidase expression may sometimes be nonspecific may reduce the specificity window. When tested in vivo, this approach was able to reduce the load of senescent cells in tissues and thus improved pathologies such as cancer and pulmonary fibrosis ${ }^{44}$, which confirms that targeted delivery of drugs into senescent cells could have therapeutic relevance. Moreover, senescence clearance could also be achieved using chemical probes ${ }^{45}$ or a cellular approach ${ }^{46}$.

Our proof-of-principle results using nanoMIPs demonstrated the feasibility of a targeted approach to detect and kill senescent cells. Previously, it has been shown that nanoMIPs can also eliminate cancer cells ${ }^{26}$. Our system opens the possibility of using tools that do not need to enter the cells to highlight and clear them, thus providing the basis of devising the first specific drug-delivery system based on the senescent surfaceome. This idea could be tested next using antibody-drug conjugates instead of nanoparticles, which could have a more immediate clinical application due to the previous experience with similar tools in approved cancer treatments ${ }^{47}$. 


\section{MATERIALS AND METHODS}

Fluorescent tagging of nanoMIP. The procedure to synthesize and analyse nanoMIPs has been adapted from previously reported protocols ${ }^{23}$ (see Supplemntary Information). NanoMIPs were tagged using two different approaches: (i) a fluorescein-based monomer (1.9 mg) was added to the polymeric mixture prior to the initiation of the polymerisation; (ii) nanoMIPs bearing primary amine groups were conjugated with NHS-dervatised fluorophores, such as Alexa Fluor 647 or DyLight 800. In particular, 20 g of either AlexaFluor 647 NHS or DyLight 800 NHS were added to $0.3 \mathrm{mg}$ of nanoMIPs in $2 \mathrm{ml}$ of phosphate buffer $\mathrm{pH}$ 7.6. After 30 minutes, the excess of NHS-dye was washed off with $15 \mathrm{ml}$ of MilliQ water using a $10 \mathrm{kDa}$ Amicon centrifuge filter. This procedure was repeated 6 times, until negligible fluorescence was detected in the eluate from the filter. Measurements of fluorescence intensity were performed using a Cary Eclipse spectrofluorometer (Varian Australia Pty Ltd) at $25{ }^{\circ} \mathrm{C}$. Excitation and emission wavelengths were set at $492 \mathrm{~nm}$ and $515 \mathrm{~nm}$. The fluorescence measurements were performed using $1 \mathrm{ml}$ quartz cuvettes of $10 \mathrm{~mm}$ path length. Before measuring fluorescence, the absorbance of each sample at the excitation wavelength was checked to be below $0.1 \mathrm{AU}$, to minimize inner filtering effects.

Cell viability. Cell viability was assessed by flow cytometry using Propidium Iodide staining, as previously described ${ }^{48}$. Samples were analysed using a BD FACSCanto II (Becton Dickenson Biosciences). Data acquisition and cell cycle analysis were done using the FACS Diva $^{\mathrm{TM}}$ version 6.1 .3 software (BD Biosciences) and graphs plotted using the GraphPad Prism 7.0 software. Alternatively, the MTS (3-(4,5-dimethylthiazol-2-yl)-5-(3- 
carboxymethoxyphenyl)-2-(4-sulfophenyl)-2H-tetrazolium) assay was used to determine the number of metabolically active cells (CellTiter 96®) AQueous One Solution Cell Proliferation Assay, Promega), following manufacturer's instructions. 100,000 cells were seeded per well in a 24 well plate and allowed to attach overnight. The media was replaced with 500 $\mu$ l of fresh media and cells were treated for 1 hour, after which they were washed gently with warm media to remove unbound MIPs and excess drug. Plates were read at an absorbance of 492nm using the Infinite F50 absorbance plate reader (Tecan) and data recorded with the Magellan for F50 data analysis software. Using Microsoft Excel 2013, cell viability was calculated by the formula: \% Viability $=($ OD Sample $) /($ OD Control $) \times 100$, where OD is the Optical Density (absorbance).

In vivo fluorescent imaging. $1.9 \mathrm{mg} / \mathrm{kg}$ of $\mathrm{B} 2 \mathrm{M}$-targeted targeted nanoMIPs were injected via the tail vein two hours before imaging. Wild type C57/BL6J mice, ranging in age from 2 to 24 months, were anaesthetised using $5 \%$ isoflurane in oxygen and maintained on $1-2 \%$ isoflurane for imaging. Prior to imaging, hair was removed from the back using hair clippers and depilatory cream. Sterile eye lubricant was applied to each eye. Mice were transferred to an IVIS Spectrum imaging system (PerkinElmer Inc) for imaging. A range of excitation and emission filters were chosen for each fluorophore to enable spectral unmixing of the fluorescence signal from background auto-fluorescence signal. 2D epi-fluorescence images were acquired from all mice. In a subset of representative mice, 3D fluorescence imaging tomography (FLIT) and whole-body micro-CT images were acquired using the IVIS Spectrum and QuantumFX micro-CT scanner (PerkinElmer Inc) respectively, to allow localisation of the signal source. Analysis was performed in LivingImage software (version 4.5.2, PerkinElmer Inc). For analysis of 2D images, whole abdomen regions were drawn on all mice and total fluorescence signal was quantified in units of radiant efficiency. The spectral unmixing tool 
was used to separate the fluorophore signal from background signal in all mice. 3D FLIT images were reconstructed and co-registered with both the LivingImage organ atlas and whole body micro-CT images using the multi-modality registration tool.

In vivo toxicity measurements. 2 animals per group were treated with nanoMIPs $(0.4 \mathrm{mg} / \mathrm{ml})$ intravenously ( $5 \mathrm{ml} / \mathrm{kg}$ body weight), intraperitoneally or by oral gavage (10 $\mathrm{ml} / \mathrm{kg}$ both). The weight of animals was measured and recorded daily for a period of 2 weeks, together with distress symptoms checks (skin appearance, natural behaviour, food and water intake, hydration status, body changes, locomotion, muscle tone). At the end of the study, animals were sacrificed and blood samples were collected through exsanguination via caudal vena cava. Blood samples were incubated on ice for 30 min to coagulate and were centrifuged for $15 \mathrm{~min}$ at 5000rpm to separate the serum. Serum samples were frozen and assayed within 2 weeks for Blood Urea Nitrogen (BUN), Alanine Transaminase (ALT) and Aspartate Transaminase (AST). Colorimetric determination of mentioned above enzyme levels was performed following manufacturer's instructions, as follows: BUN (Kit No. EIABUN, Invitrogen, Carlsbad, CA), AST (K753-100, Biovision, Minneapolis, MN, USA) and ALT (Kit No. 700260, Cayman chemicals, Michigan, USA). Every serum sample was assayed in triplicates for all tested enzymes. For analysis of kidney and liver enzymes activity, the statistical analyses were performed using One-way ANOVA (GraphPad Software, La Jolla, CA). Differences were considered significant at the 95\% level of confidence. 


\section{ACKNOWLEDGEMENTS}

Work in SM's lab was supported by an Innovation Fellowship from the University of Leicester

and the M.C. Andreu Memorial Fund. AEEA was partly supported by a TETFund PhD fellowship. MP was supported by an MIBTP fellowship and the M. C. Andreu PhD Support Fellowship. MA was supported by a Saudi Government Doctoral Scholarship. We thank Dr Andrew Jamieson for help in designing the epitope for nanoMIP synthesis. We thank Dr Kees Straatman and the Advanced Imaging Facility for their help in obtaining and preparing the images. We also thank the staff of the Division of Biomedical Services, University of Leicester, for their care of the experimental animals.

\section{AUTHORS CONTRIBUTIONS}

Experiments were designed by AEEA, FC, SP and SM. nanoMIPs were generated by FC, SP, BHA and EP. Cellular experiments were performed by AEA with help from MP, MC and LC. Mouse work was performed by AEA with help from GKF and MP. JJ and MEK contributed to the mouse imaging experiments and analysis. The manuscript was written by SM with help from AEEA and FC, and reviewed by all authors.

\section{COMPETING INTERESTS}

FC and SP declare competing financial interests: FC is an employee and SP is a founder of MIP Diagnostics Ltd. 


\section{REFERENCES}

1 Collado, M. \& Serrano, M. Senescence in tumours: evidence from mice and humans. Nature reviews. Cancer 10, 51-57, doi:10.1038/nrc2772 (2010).

2 Campisi, J. \& d'Adda di Fagagna, F. Cellular senescence: when bad things happen to good cells. Nature reviews. Molecular cell biology 8, 729-740, doi:10.1038/nrm2233 (2007).

3 Kuilman, T., Michaloglou, C., Mooi, W. J. \& Peeper, D. S. The essence of senescence. Genes \& development 24, 2463-2479, doi:10.1101/gad.1971610 (2010).

4 Chen, Z. et al. Crucial role of p53-dependent cellular senescence in suppression of Ptendeficient tumorigenesis. Nature 436, 725-730 (2005).

5 Michaloglou, C. et al. BRAFE600-associated senescence-like cell cycle arrest of human naevi. Nature 436, 720-724 (2005).

6 Collado, M. et al. Tumour biology: senescence in premalignant tumours. Nature 436, 642, doi:10.1038/436642a (2005).

7 Drummond-Barbosa, D. Stem Cells, Their Niches and the Systemic Environment: An Aging Network. Genetics 180, 1787-1797, doi:DOI 10.1534/genetics.108.098244 (2008).

8 Krizhanovsky, V. et al. Senescence of activated stellate cells limits liver fibrosis. Cell 134, 657-667, doi:10.1016/j.cell.2008.06.049 (2008).

9 Kang, T. W. et al. Senescence surveillance of pre-malignant hepatocytes limits liver cancer development. Nature 479, 547-551, doi:10.1038/nature10599 (2011).

10 Munoz-Espin, D. \& Serrano, M. Cellular senescence: from physiology to pathology. Nature reviews. Molecular cell biology 15, 482-496, doi:10.1038/nrm3823 (2014).

11 He, S. \& Sharpless, N. E. Senescence in Health and Disease. Cell 169, 1000-1011, doi:10.1016/j.cell.2017.05.015 (2017).

12 Munoz-Espin, D. et al. Programmed cell senescence during mammalian embryonic development. Cell 155, 1104-1118, doi:10.1016/j.cell.2013.10.019 (2013).

13 Demaria, M. et al. An essential role for senescent cells in optimal wound healing through secretion of PDGF-AA. Dev Cell 31, 722-733, doi:10.1016/j.devcel.2014.11.012 (2014).

14 Campisi, J. Senescent cells, tumor suppression, and organismal aging: good citizens, bad neighbors. Cell 120, 513-522 (2005).

15 Lawless, C. et al. Quantitative assessment of markers for cell senescence. Experimental gerontology 45, 772-778, doi:10.1016/j.exger.2010.01.018 (2010).

16 Matjusaitis, M., Chin, G., Sarnoski, E. A. \& Stolzing, A. Biomarkers to identify and isolate senescent cells. Ageing research reviews 29, 1-12, doi:10.1016/j.arr.2016.05.003 (2016).

17 Serrano, M., Lin, A. W., McCurrach, M. E., Beach, D. \& Lowe, S. W. Oncogenic ras provokes premature cell senescence associated with accumulation of p53 and p16INK4a. Cell 88, 593-602 (1997).

18 Dimri, G. P. et al. A Biomarker That Identifies Senescent Human-Cells in Culture and in Aging Skin in-Vivo. Proceedings of the National Academy of Sciences of the United States of America 92, 9363-9367, doi:DOI 10.1073/pnas.92.20.9363 (1995).

19 Yang, N. C. \& Hu, M. L. The limitations and validities of senescence associated-betagalactosidase activity as an aging marker for human foreskin fibroblast Hs68 cells. Experimental gerontology 40, 813-819, doi:10.1016/j.exger.2005.07.011 (2005). 
20 Macip, S. et al. Inhibition of p21-mediated ROS accumulation can rescue p21-induced senescence. The EMBO journal 21, 2180-2188, doi:10.1093/emboj/21.9.2180 (2002).

21 Althubiti, M. et al. Characterization of novel markers of senescence and their prognostic potential in cancer. Cell death \& disease 5, e1528, doi:10.1038/cddis.2014.489 (2014).

22 Althubiti, M. \& Macip, S. Detection of Senescent Cells by Extracellular Markers Using a Flow Cytometry-Based Approach. Methods in molecular biology 1534, 147-153, doi:10.1007/978-1-4939-6670-7_14 (2017).

23 Poma, A. et al. Solid-Phase Synthesis of Molecularly Imprinted Polymer Nanoparticles with a Reusable Template - "Plastic Antibodies". Advanced functional materials 23, 2821-2827, doi:10.1002/adfm.201202397 (2013).

24 Canfarotta, F., Poma, A., Guerreiro, A. \& Piletsky, S. Solid-phase synthesis of molecularly imprinted nanoparticles. Nature protocols 11, 443-455, doi:10.1038/nprot.2016.030 (2016).

25 Gussow, D. et al. The human beta 2-microglobulin gene. Primary structure and definition of the transcriptional unit. Journal of immunology 139, 3132-3138 (1987).

26 Canfarotta, F. et al. Specific Drug Delivery to Cancer Cells with Double-Imprinted Nanoparticles against Epidermal Growth Factor Receptor. Nano letters 18, 4641-4646, doi:10.1021/acs.nanolett.7b03206 (2018).

27 Salama, R., Sadaie, M., Hoare, M. \& Narita, M. Cellular senescence and its effector programs. Genes \& development 28, 99-114, doi:10.1101/gad.235184.113 (2014).

28 Baker, D. J. et al. Naturally occurring p16(Ink4a)-positive cells shorten healthy lifespan. Nature 530, 184-189, doi:10.1038/nature16932 (2016).

29 Baker, D. J. et al. Clearance of p16Ink4a-positive senescent cells delays ageingassociated disorders. Nature 479, 232-236, doi:10.1038/nature10600 (2011).

30 Childs, B. G. et al. Senescent cells: an emerging target for diseases of ageing. Nature reviews. Drug discovery, doi:10.1038/nrd.2017.116 (2017).

31 Canfarotta, F. W., A.; Sadler, R; McGill, P.; Guerreiro, A.; Papkovsky, D.; Haupt, K.; Piletsky, S. Biocompatibility and Internalization of Molecularly Imprinted Nanoparticles. Nano Research In press (2016).

32 Kirkland, J. L. \& Tchkonia, T. Cellular Senescence: A Translational Perspective. EBioMedicine 21, 21-28, doi:10.1016/j.ebiom.2017.04.013 (2017).

33 Hoshino, Y. et al. Recognition, neutralization, and clearance of target peptides in the bloodstream of living mice by molecularly imprinted polymer nanoparticles: a plastic antibody. Journal of the American Chemical Society 132, 6644-6645, doi:10.1021/ja102148f (2010).

34 Saffrey, M. J. Aging of the mammalian gastrointestinal tract: a complex organ system. Age 36, 9603, doi:10.1007/s11357-013-9603-2 (2014).

35 Biran, A. et al. Quantitative identification of senescent cells in aging and disease. Aging Cell 16, 661-671, doi:10.1111/acel.12592 (2017).

36 Korposh, S. et al. Selective vancomycin detection using optical fibre long period gratings functionalised with molecularly imprinted polymer nanoparticles. The Analyst 139, 2229-2236, doi:10.1039/c3an02126b (2014).

37 Canfarotta, F. et al. A novel thermal detection method based on molecularly imprinted nanoparticles as recognition elements. Nanoscale 10, 2081-2089, doi:10.1039/c7nr07785h (2018).

38 Basozabal, I., Guerreiro, A., Gomez-Caballero, A., Aranzazu Goicolea, M. \& Barrio, R. J. Direct potentiometric quantification of histamine using solid-phase imprinted nanoparticles as recognition elements. Biosens Bioelectron 58, 138-144, doi:10.1016/j.bios.2014.02.054 (2014). 
39 Caceres, C. et al. Does size matter? Study of performance of pseudo-ELISAs based on molecularly imprinted polymer nanoparticles prepared for analytes of different sizes. The Analyst 141, 1405-1412, doi:10.1039/c5an02018b (2016).

40 Canfarotta, F., Smolinska-Kempisty, K. \& Piletsky, S. Replacement of Antibodies in Pseudo-ELISAs: Molecularly Imprinted Nanoparticles for Vancomycin Detection. Methods in molecular biology 1575, 389-398, doi:10.1007/978-1-4939-6857-2_25 (2017).

41 Smolinska-Kempisty, K. et al. A comparison of the performance of molecularly imprinted polymer nanoparticles for small molecule targets and antibodies in the ELISA format. Scientific reports 6, 37638, doi:10.1038/srep37638 (2016).

42 Cecchini, A. et al. In Vivo Recognition of Human Vascular Endothelial Growth Factor by Molecularly Imprinted Polymers. Nano letters 17, 2307-2312, doi:10.1021/acs.nanolett.6b05052 (2017).

43 Agostini, A. et al. Targeted cargo delivery in senescent cells using capped mesoporous silica nanoparticles. Angew Chem Int Ed Engl 51, 10556-10560, doi:10.1002/anie.201204663 (2012).

44 Munoz-Espin, D. et al. A versatile drug delivery system targeting senescent cells. EMBO molecular medicine, doi:10.15252/emmm.201809355 (2018).

45 Lozano-Torres, B. et al. An OFF-ON Two-Photon Fluorescent Probe for Tracking Cell Senescence in Vivo. Journal of the American Chemical Society 139, 8808-8811, doi:10.1021/jacs.7b04985 (2017).

46 Kim, K. M. et al. Identification of senescent cell surface targetable protein DPP4. Genes \& development 31, 1529-1534, doi:10.1101/gad.302570.117 (2017).

47 Thomas, A., Teicher, B. A. \& Hassan, R. Antibody-drug conjugates for cancer therapy. Lancet Oncol 17, e254-e262, doi:10.1016/S1470-2045(16)30030-4 (2016).

48 Samuel, J. et al. Posttranscriptional Upregulation of p53 by Reactive Oxygen Species in Chronic Lymphocytic Leukemia. Cancer research 76, 6311-6319, doi:10.1158/0008-5472.CAN-16-0843 (2016). 


\section{FIGURE LEGENDS}

Figure 1. Generation of nanoMIPs. (A) Crystal structure (left) and surface presentation (right) of B2M, with the epitope used for nanoMIP design, near the C-terminal, indicated in magenta. Structure adapted using PyMOL 1.0. (B) Representative dynamic light scattering images of the B2M nanoMIPs. Scale bars are, respectively, $1 \mu \mathrm{m}$ and $200 \mathrm{~nm}$.

Figure 2. Fluorescent nanoMIPs against B2M selectively bind to senescent cells. (A) Representative Western blot showing B2M protein expression in EJp16 cells, with $\beta$-actin as a loading control. Senescence was induced by culturing cells without tetracycline for 4 days. (B) FACS analysis of EJp16 and incubated with B2M-targeted fluorescent nanoMIPs. Numbers show the percentage of cells positive for green fluorescence above background levels, which is indicative of cells to which the nanoMIPs attached. (C) Representative images of fluorescent microscopy taken 4 hours after EJp16 were incubated with the nanoMIPs. Fluorescent particles in each microscopic field were quantified using Image $J$ software. Results show mean \pm SD of triplicate independent experiments. ${ }^{* *}: \mathrm{p}<0.005$ (two-tailed unpaired t test). (C) Representative image of fluorescent microscopy from the same cells, showing that nanoMIPs bind to the membrane of senescent EJp16.

Figure 3. Targeted delivery of drugs into senescent cells by nanoMIPs. (A) Colony Formation assays of control EJp16 (cultured in the presence of tet) incubated with nanoMIPs. Graphs show results from two independent experiments in duplicates, and are plotted as mean \pm SD. (B) Representative cell cycle analysis plots obtained by FACS analysis of PI-stained EJp16 cells incubated with B2M nanoMIPs for 24 hours. Percentage of cell death (subG 1 populations) are indicated. (C) Representative confocal microscope images of control and 
senescent EJp16 fixed with formalin and stained with DAPI 24 hours after incubation with B2M nanoMIPs at $37^{\circ} \mathrm{C}$. (D) Percentage of cell death, as measured by PI staining, of Control and Senescent EJp16 treated with different concentrations of oligomycin, gramicidin, ABT263, quercetin and dasatinib for 48 hours. Results represent the average of three independent experiments. Error bars show standard deviation. (E) Cell viability measured by an MTS assay of proliferating and senescent EJp16 (4 days after tet removal) treated with B2M nanoMIPs, $10 \mu \mathrm{M}$ Dasatinib-conjugated B2M nanoMIPs or $10 \mu \mathrm{M}$ Dasatinib for 24 hours. Results show average $\pm \mathrm{SD}$ of four replicates. $*$ : $\mathrm{p}<0.05$; ****: $\mathrm{p}<0.0005$; ns: not significant (two-tailed paired t tests).

Figure 4. Effects of nanoMIPs in vivo. (A) Body weight change of mice exposed to a nanoMIP solution $(0.4 \mathrm{mg} / \mathrm{ml})$ via 3 different means of delivery: by oral gavage $(10 \mathrm{ml} / \mathrm{kg})$, intraperitoneally $(10 \mathrm{ml} / \mathrm{kg})$ and intravenously $(5 \mathrm{ml} / \mathrm{kg})$. The weight of two animals was measured 14 days after treatment and normalized to the weight on day 0 . Error bars show \pm SEM. (B) Blood Urea Nitrogen (BUN), Alanine aminotransferase (ALT), and Aspartate aminotransferase (AST) levels in serum samples from two mice 14 days after treatment with nanoMIPs. Blood samples were collected through exsanguination via caudal vena cava and every sample was assayed in triplicates for all tested enzymes. Data represent the mean \pm SD. The statistical analysis revealed nonsignificant difference between all the groups and the control (One-way ANOVA, p>0.05).

Figure 5. In vivo imaging of senescent cells by fluorescent nanoMIPs. (A) Representative 2D epi-fluorescence images of young (2 month) and old (11 month) mice injected intravenously with DyLight ${ }^{\mathrm{TM}}$ 800-labelled B2M nanoMIPs, compared with untreated controls 
from same age group. (B) A 3D registration and signal localization of these nanoMIPs in a 16 month old mouse in the abdominal cavity. (C) Ex vivo imaging of dissected young (2 month) and old (11 month) mice in supine position. The first two animals of each group were given B2M nanoMIPs, while the last one only received the vehicle (to control for autofluorescence of the tissues). Total radiant efficiency of fluorescence signals of the nanoMIP-treated mice is quantified and presented as mean \pm SD. Unpaired t test shows statistical significance (*: $\mathrm{p}<0.05)$. 
$A$
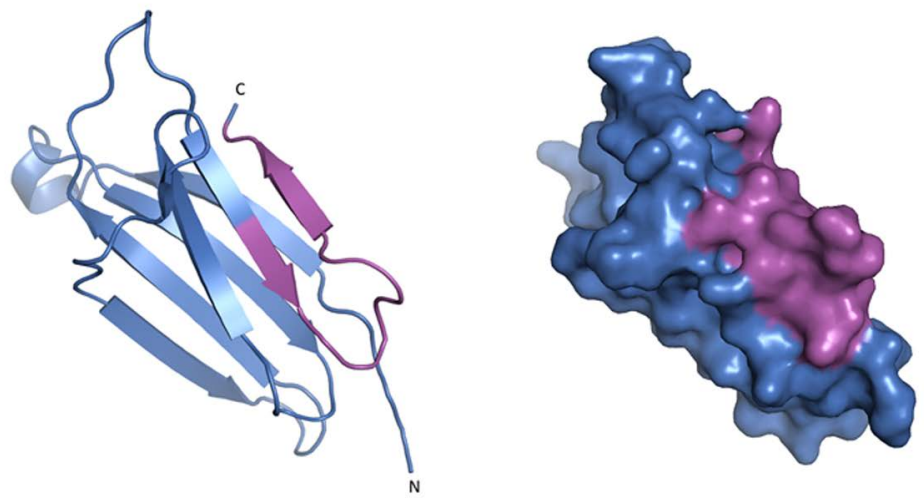

B
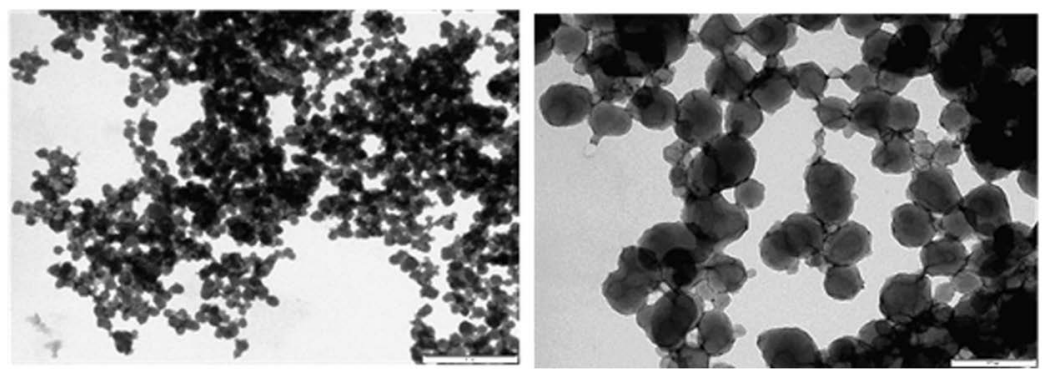

Figure 1 


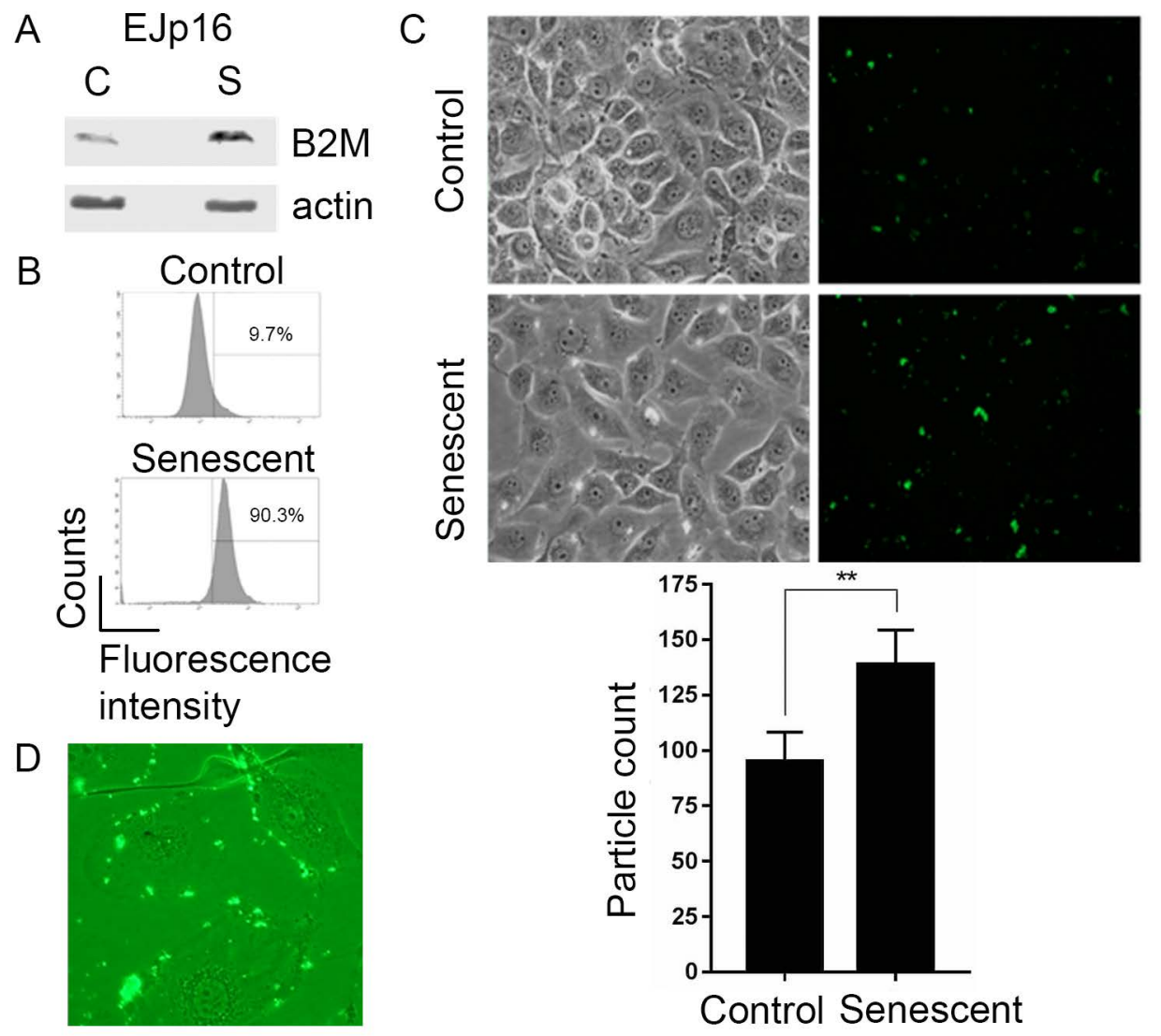

Figure 2 
$A$

Control +nanoMIPS
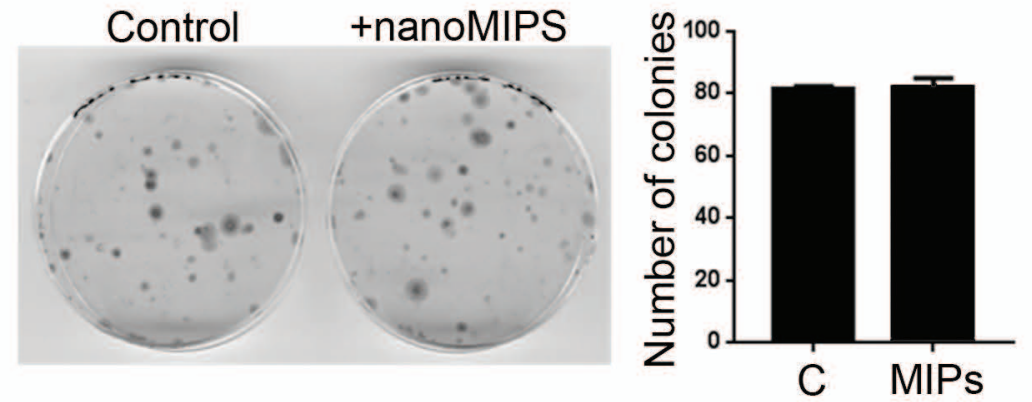

C

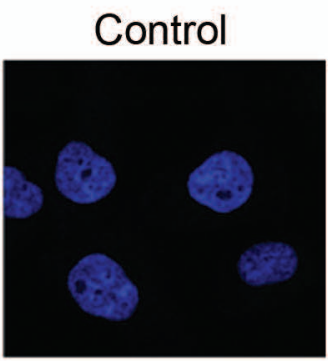

Senescent

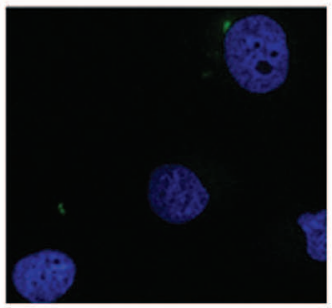

D ${ }^{80}{ }^{\text {Control }}$

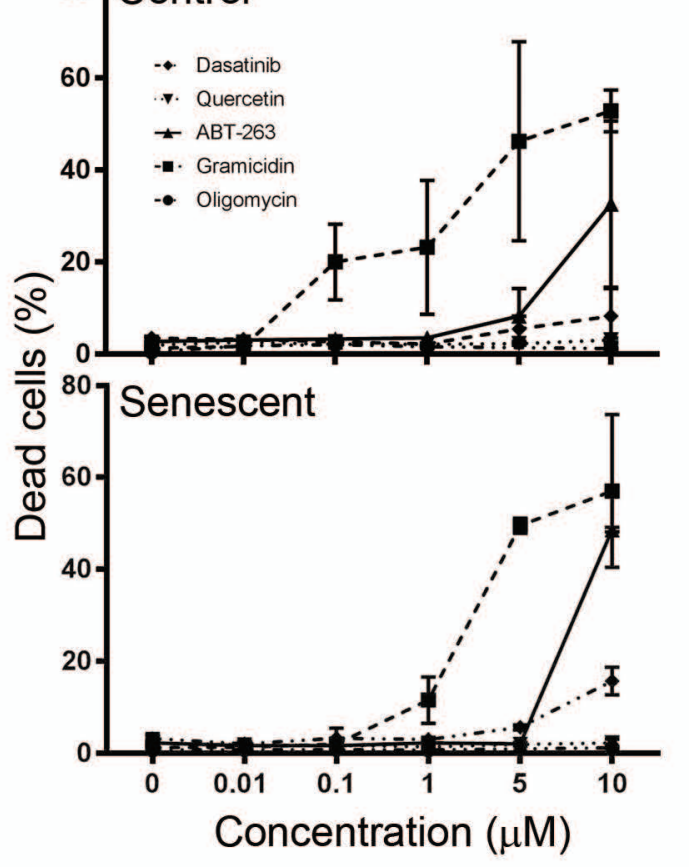

B

Control

+nanoMIPS

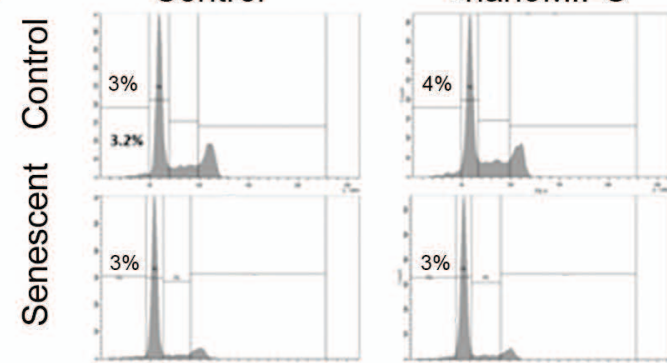

E

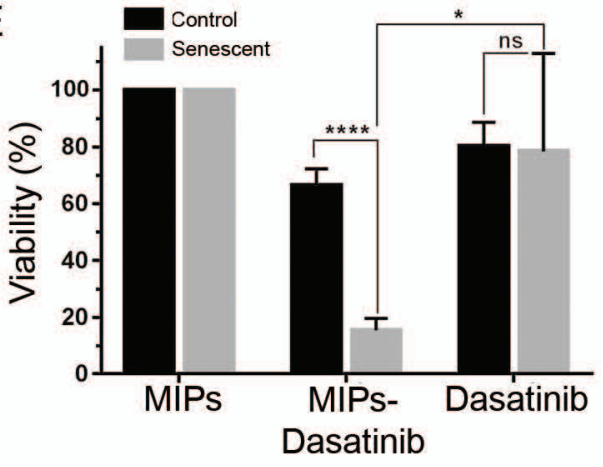

Figure 3 

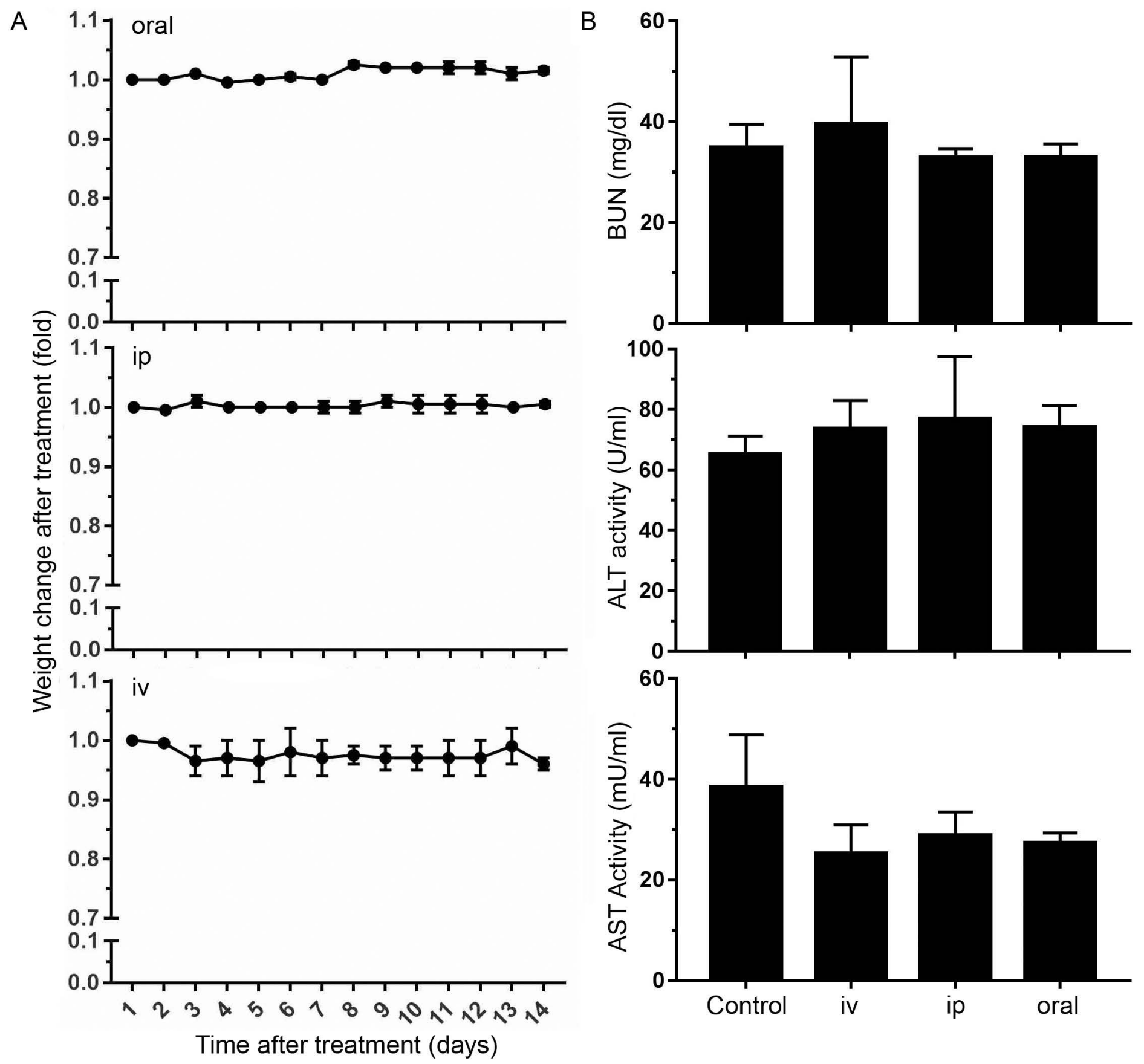

Figure 4 
A

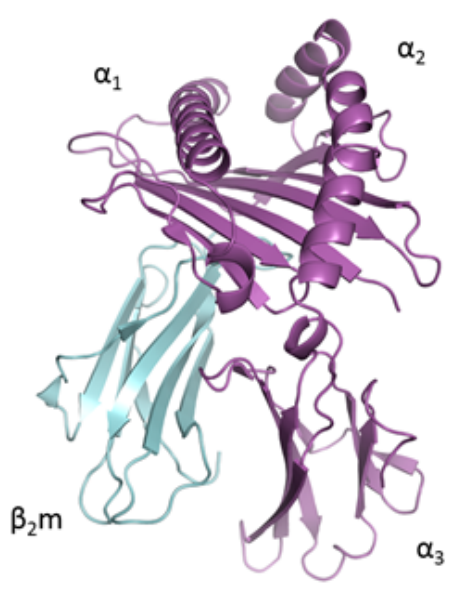

B

\author{
RVNHVTLSQPKIVKWGK Human \\ RVKHVSMAEPKTVYWDR Mouse
}

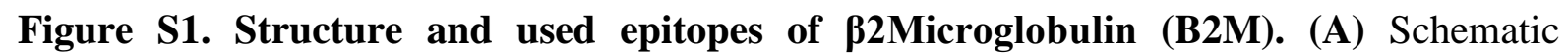
representation of the structure of the human histocompatibility antigen HLA-A2 showing the positions of the $\alpha 1, \alpha 2, \alpha 3$ and B2M domains. Structure adapted using PyMOL 1.0. (B) Homologies between the protein sequences of the B2M epitope in human and mice, using a BLAST alignment. 

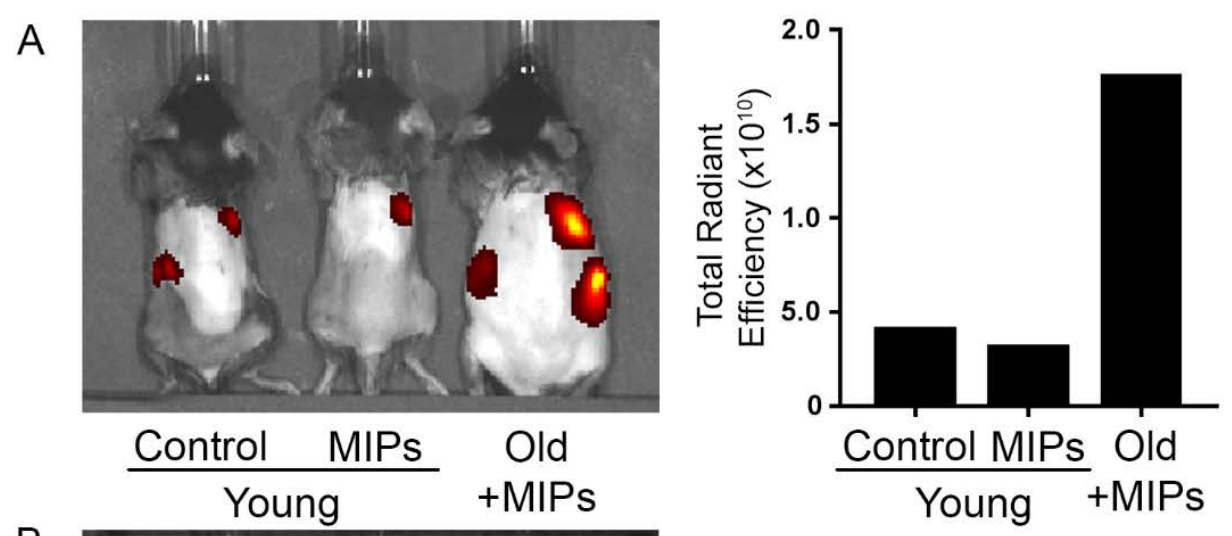

$\mathrm{B}$
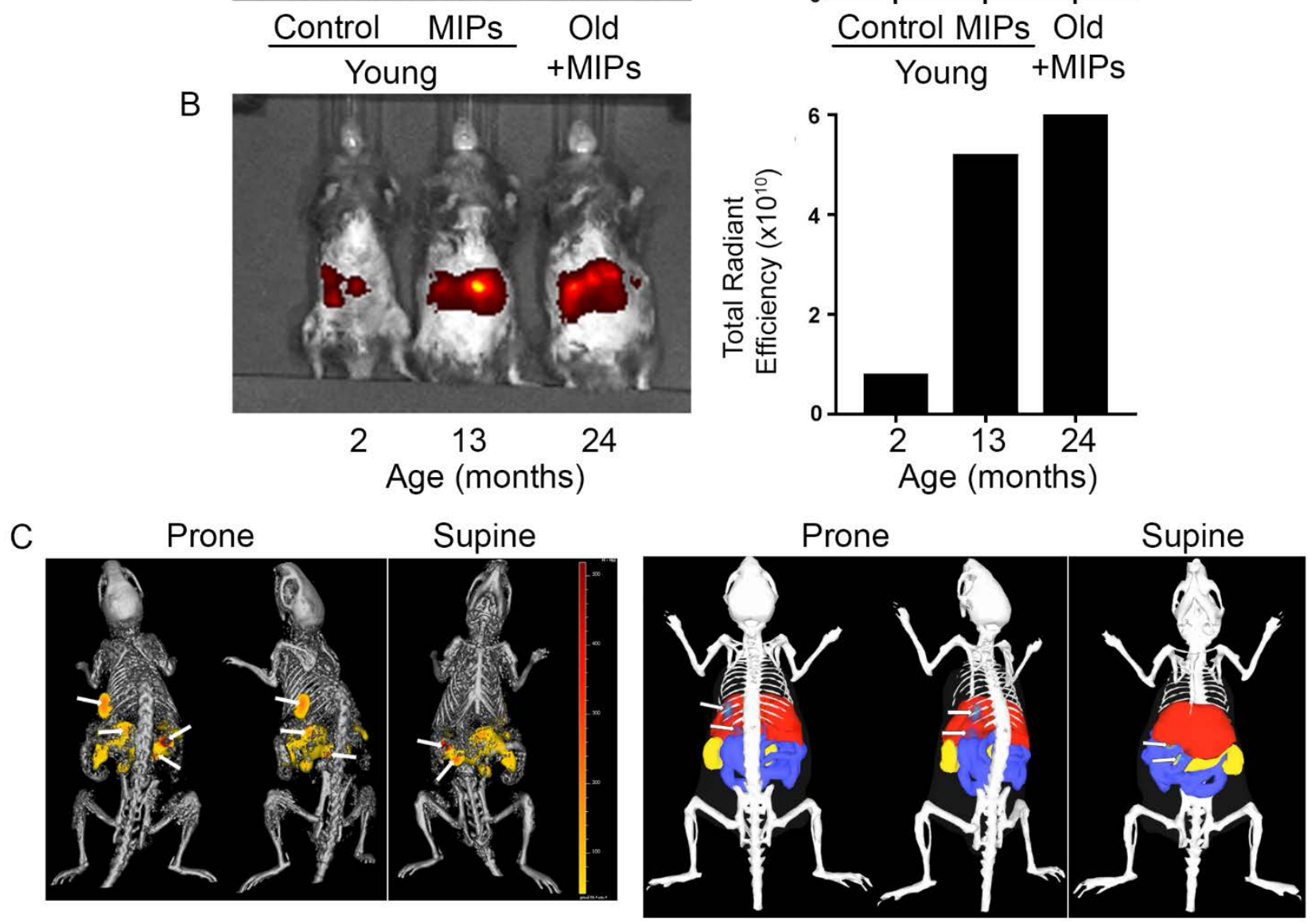

Figure S2. In vivo imaging of fluorescent B2M NanoMIPs. (A) Representative images of young (2 months) and old (15 months) mice injected intravenously with Alexa Fluor 647tagged B2M MIPs. Control mice was injected only vehicle. Animals were imaged in the prone position 2 hours after injection. Total fluorescence signals were quantified and are shown in units of radiant efficiency. (B) Same as (A), with another group of mice of different ages, imaged in supine position. (C) 3D whole-body micro-CT (top) and fluorescence imaging tomography (FLIT, bottom) of the 15 month old mouse in (A). Arrows show the localization of fluorescent signals, which map to the right lung, right kidney and jejunum areas. Of note, lung and kidney also showed background fluorescence in young an untreated mice. 


\section{SUPPLEMENTARY TABLES}

\begin{tabular}{|c|c|c|}
\hline \multicolumn{2}{|r|}{ Parameters } & Score \\
\hline Appearance & $\begin{array}{l}\text { - Normal } \\
\text { - Slight piloerection } \\
\text { - Marked piloerection } \\
\text { - Changes from normal to signs of deterioration of } \\
\text { coat and skin }\end{array}$ & $\begin{array}{c}0 \\
1.5 \\
3 \\
4-6\end{array}$ \\
\hline Natural behaviour & $\begin{array}{l}\text { - Normal } \\
\text { - Minor changes } \\
\text { - Less mobile and isolated } \\
\text { - Restless or very still }\end{array}$ & $\begin{array}{c}0 \\
1 \\
2 \\
3-6\end{array}$ \\
\hline Food and water intake & $\begin{array}{l}\text { - Normal } \\
\text { - Body weight loss }<5 \% \\
\text { - Body weight loss }<10 \%-<20 \%\end{array}$ & $\begin{array}{c}0 \\
3 \\
4-6\end{array}$ \\
\hline Hydration status & $\begin{array}{l}\text { - Normal } \\
\text { - Abnormal skin pinch test } \\
\text { - Very Abnormal skin pinch test }\end{array}$ & $\begin{array}{c}0 \\
2.5 \\
2.5-6 \\
\end{array}$ \\
\hline Body changes & $\begin{array}{l}\text { - Normal } \\
\text { - Backbones visible } \\
\text { - Backbones very visible } \\
\text { - Tachypnoea (fast breathing) } \\
\text { - Dyspnoea (difficult breathing) }\end{array}$ & $\begin{array}{l}0 \\
3 \\
6 \\
3 \\
6\end{array}$ \\
\hline Locomotion & $\begin{array}{l}\text { - Slightly abnormal gait/posture } \\
\text { - Markedly abnormal gait/posture }\end{array}$ & $\begin{array}{c}0-1.5 \\
3-6\end{array}$ \\
\hline Muscle tone & $\begin{array}{l}\text { - Muscle groups have normal tone or mass } \\
\text { - Muscle mass slightly soft } \\
\text {-Muscle mass less firm, abdomen slightly soft } \\
\text { - Muscle mass very thin, soft, undefined } \\
\text { - Muscle mass has no tone or definition }\end{array}$ & $\begin{array}{c}0 \\
2 \\
3 \\
4 \\
5-6\end{array}$ \\
\hline Specific Indicators & $\begin{array}{l}\text { - Tumour size }>1.0 \mathrm{~cm}^{3} \\
\text { - Tumour impeding movement }\end{array}$ & $\begin{array}{l}6 \\
6\end{array}$ \\
\hline
\end{tabular}

Table S1. Frailty score to assess the impact of nanoMIPs on mouse health. All mice had a total score of 0 when adding all parameters, for the duration of the study. Mice were assessed daily. 


\section{SUPPLEMENTARY METHODS}

Preparation of epitope-modified glass beads (solid-phase). Glass beads (Spheriglass ${ }^{\circledR} 2429$ CP00, 53-106 $\mu \mathrm{m}$ diameter, from Blagden Chemicals) were first activated by boiling in a $2 \mathrm{M}$ $\mathrm{NaOH}$ solution for $15 \mathrm{~min}$, then washed with deionized water and rinsed with acetone, dried at $80{ }^{\circ} \mathrm{C}$ and subsequently incubated overnight in $2 \% \mathrm{v} / \mathrm{v} 3$-aminopropyltrimethyloxysilane in dry toluene solution ( $0.4 \mathrm{~mL}$ solution/g glass beads). This last step leads to beads bearing $\mathrm{NH}_{2}$ surface groups. Afterwards, $60 \mathrm{~g}$ of glass beads were placed in a solution of succinimidyl iodoacetate linker, at $0.2 \mathrm{mg} / \mathrm{ml}$ in acetonitrile for $2 \mathrm{~h}$ in the dark $(0.4 \mathrm{ml}$ solution $\mathrm{g} / \mathrm{glass}$ beads). The succinic moiety allows the linker to react with the amine-derivatized solid-phase, and the haloacetyl group enables subsequent coupling with the thiol group from cysteine added on the N-terminus of the peptide sequence. Afterwards, the beads were washed with $400 \mathrm{ml}$ of acetonitrile in a sintered glass funnel and placed in a bottle containing $7 \mathrm{mg}$ of the cysteinemodified peptide epitope (primary template) in $40 \mathrm{ml}$ of deoxygenated phosphate buffered saline (PBS) containing $5 \mathrm{mM}$ EDTA, $\mathrm{pH}$ 8.2. For the preparation of control nanoMIPs, biotin was used as a template. In particular, $60 \mathrm{~g}$ of amine-derivatized glass beads were incubated with a solution of biotin (Sigma Aldrich) containing 1-ethyl-3-(3-dimethylaminopropyl) carbodiimide hydrochloride (EDC) and N-hydroxysuccinimide (NHS) (both from Sigma Aldrich) at 0.5, 10 and $15 \mathrm{mg} / \mathrm{ml}$ respectively, in PBS, pH 7.4. After overnight incubation, the beads were washed with $500 \mathrm{ml}$ of water in a sintered funnel and used for the synthesis of nanoMIPs. After overnight incubation, the beads were washed with $500 \mathrm{ml}$ of water in a sintered funnel and used for the synthesis of nanoMIPs.

Synthesis of nanoMIPs. The following monomers were dissolved in PBS 5 mM, pH 7.4 (100 ml): N-isopropylacrylamide (39 mg), N,N'-methylenebisacrylamide (4 mg), N-tert- 
butylacrylamide (33 mg), N-(3-aminopropyl)methacrylamide hydrochloride (5.8 mg), acrylic acid $(2.2 \mu \mathrm{l})$ and $\mathrm{N}$-fluoresceinylacrylamide (2.9 mg). N-tert-butylacrylamide was previously dissolved in ethanol $(0.5 \mathrm{ml})$ and then added to the aqueous solution. For synthesis of control nanoMIPs (non-B2M-imprinted), biotin-derivatized glass beads were used, and all remaining conditions were kept identical. This was necessary, as no non-imprinted materials can be produced using the solid-phase approach (because the affinity separation step cannot be performed in the absence of template, as the majority of the material produced is eluted during washing steps). Biotin is sufficiently dissimilar to the B2M epitope and not naturally exposed on the cell surface, so these can be considered for practical purposes as non-B2M-imprinted materials. The solution containing the monomers was degassed under vacuum and sonicated for $5 \mathrm{~min}$, and then purged with $\mathrm{N}_{2}$ for $20 \mathrm{~min}$. Then, $60 \mathrm{~g}$ of B2M-derivatized glass beads were added to the solution. Polymerization was initiated by adding ammonium persulfate aqueous solution $(800 \mu \mathrm{l}, 60 \mathrm{mg} / \mathrm{ml})$ and $\mathrm{N}, \mathrm{N}, \mathrm{N}^{\prime}, \mathrm{N}^{\prime}$-tetramethylethylenediamine $(24 \mu \mathrm{l})$. The headspace was flushed with $\mathrm{N}_{2}$ and the bottle sealed with a screw cap. Polymerization was carried-out at room temperature for $1 \mathrm{~h}$. Subsequently, the content of the polymerization vessel was poured into a solid-phase extraction (SPE) cartridge $(60 \mathrm{ml})$ equipped with a frit $(20 \mu \mathrm{m}$ porosity). A total of 9 washes with $20 \mathrm{ml}$ of distilled water at $20^{\circ} \mathrm{C}$ was carried out to remove low affinity nanoMIPs, polymer and unreacted monomer. Afterwards, the SPE cartridge containing the solid-phase was placed in a water bath at $70{ }^{\circ} \mathrm{C}$ for $15 \mathrm{~min}$. An aliquot of $20 \mathrm{ml}$ of distilled water pre-warmed at $65{ }^{\circ} \mathrm{C}$ was poured into the SPE to collect the high-affinity nanoMIPs. This action was repeated 7 times, until about $140 \mathrm{ml}$ of a solution of high-affinity nanoMIPs in water were collected. To ensure complete removal of all unreacted monomer from the bulk of the nanoparticles, aliquots of the collected solution were concentrated down to $2 \mathrm{ml}$ using a centrifugal dialysis cartridge fitted with a membrane with $30 \mathrm{kDa}$ molecular weight cut-off (Amicon Ultra, Merck Millipore). This was followed by seven washes (14 ml) with 
deionized water on the same dialysis cartridge. The procedure was repeated using biotinderivatized glass beads to prepare control nanoMIPs. NanoMIPs were tested by surface plasmon resonance (SPR) to determine their affinity towards the primary template and compared against control nanoparticles (non-imprinted) prepared with the same monomer composition. An equilibrium dissociation constant (KD) of $52 \mathrm{nM}$ was obtained for B2Mimprinted nanoMIPs.

Size and concentration analysis of nanoMIPs. Particle size was measured with a Zetasizer Nano (Nano-S) particle-size analyzer from Malvern Instruments Ltd (UK). An aliquot of the dispersion of nanoMIPs in distilled water was sonicated for 2 min and then analyzed by dynamic light scattering at $25{ }^{\circ} \mathrm{C}$ in a $3 \mathrm{~cm}^{3}$ disposable polystyrene cuvette. Attenuator position, measurement duration and number of runs were automatically chosen by the instrument. The values are reported as an average of 4 measurements. TEM images of nanoMIPs were taken using a JEOL JEM 1010, $100 \mathrm{kV}$ high contrast TEM equipped with a Gatan SC1000 Orius CCD camera (Gatan, Abingdon Oxon, UK). Samples for the analysis have been prepared by depositing a drop of the nanoMIPs dispersion, previously filtered through a $1.2 \mu \mathrm{m}$ PES syringe filter, on a carbon-coated TEM copper grid (400 mesh), and leaving them to dry at room temperature. The nanoMIP concentration was assessed by freezedrying an aliquot $(20 \mathrm{ml})$ of the particle solution and then weighing the solid.

Surface plasmon resonance (SPR) analysis. Experiments were performed on SIA Au SPR gold chips (GE Healthcare) modified with mercaptoundecanoic acid. Bare gold chips were first cleaned by hydrogen plasma at $50 \mathrm{~W}$ during five minutes on an Emitech K1050X Plasma Cleaner (Emitech) and then placed in ethanol containing $2.2 \mathrm{mg} / \mathrm{ml}$ mercaptoundecanoic acid, overnight in a sealed vial. After surface modification, chips were rinsed with ethanol and dried under a stream of N2, assembled on the holder following the manufacturer instructions and 
docked onto the SPR instrument (Biacore 3000, GE Healthcare). For polymer coupling, the chips were activated by injection of $100 \mu \mathrm{l}$ EDC $0.2 \mathrm{M}$ and NHS $0.05 \mathrm{M}$ in water at $5 \mu \mathrm{l} / \mathrm{min}$, followed by 1 injection of nanoMIPs (at $0.1 \mathrm{mg} / \mathrm{ml}$ ) in phosphate buffer at $5 \mu \mathrm{l} / \mathrm{min}$ until around 1000 RU were reached. Remaining NHS esters were deactivated by injection of $100 \mu \mathrm{l}$ of ethanolamine hydrochloride $(0.1 \mathrm{M})$ at $10 \mu \mathrm{l} / \mathrm{min}$ in PBS. The B2M epitope was then injected onto the nanoMIP-modified chip in concentrations ranging from 0.01 to $50 \mathrm{nM}$, and a control channel passivated with ethanolamine was used as a control. Analysis was performed in $1 \times$ PBS at pH 7.4. Kinetic analysis of the sensorgrams was performed with the BiaEvaluation software v4.1 assuming a 1:1 Langmuir binding model with baseline drifting.

Cell culture and senescence induction. All cells were cultured in Dulbecco's Modified Eagle’s Medium (DMEM) supplemented with 10\% Foetal Bovine Serum (FBS), 100 IU/ml penicillin and $100 \mu \mathrm{g} / \mathrm{ml}$ streptomycin. Cells were incubated at $37^{\circ} \mathrm{C}$ in a humidified incubator with $5 \% \mathrm{CO}_{2}$. To keep the EJ cells proliferating, $1 \mu \mathrm{M}$ tetracycline was added to the culture media and this was replaced every three days. EJ p16 cells were maintained in complete culture media supplemented with $100 \mu \mathrm{g} / \mathrm{ml}$ hygromycin and $2 \mu \mathrm{g} / \mathrm{ml}$ puromycin. To induce senescence by tetracycline removal, cells were trypsinized, washed with $1 \mathrm{x}$ phosphate buffered saline (PBS) and centrifuged at 1,100 rpm for three minutes. This wash step was carried out three times. Cells were incubated with quercetin (Selleckchem), dasatinib (Selleckchem), oligomycin (Selleckchem), gramicidin (Sigma) or ABT-263 (Selleckchem) for the specified times.

Western Blot Analysis. For lysate extraction, the medium was removed from cells and plates were washed once with 1x PBS. Cells were trypsinized, collected, centrifuged and the pellets kept on ice. The cell pellet was re-suspended in $100 \mu \mathrm{l}$ of ice cold RIPA lysis buffer (radio 
immunoprecipitation assay - 150mM NaCl, 50mM Tris $\mathrm{HCl} \mathrm{pH}$ 8.0, 1\% NP40, 0.1\% SDS, $0.5 \%$ sodium deoxycholate) containing a 1:100 dilution of protease inhibitor cocktail and 1:100 dilution of phosphatase inhibitor cocktail (Sigma Aldrich) and incubated for 20 minutes on ice. Cells were ruptured by passing through a syringe 10 times or with sonication, and centrifuged at $14,000 \mathrm{~g}$ for 15 minutes at $4^{\circ} \mathrm{C}$. The supernatant was transferred into Eppendorf tubes and protein concentrations were determined using the Bradford protein assay (Fermentas). $35 \mu 1$ of 4X Laemmli buffer was added and samples were heated at $95^{\circ} \mathrm{C}$ for 5 minutes. $20 \mu \mathrm{g}$ of total protein per sample were subjected to $10 \%$ or $15 \%$ SDS-PAGE and transferred to ImmobilonP membranes (Millipore). Primary antibodies used were: $\beta$-actin (Abcam, \#ab8227), p16 (Abcam, \#ab54210) and B2M (LifeSpan BioSciences, \#LS-B2200 and Abcam, \#Ab87483).

Fluorescence measurements. Cells were grown on sterile $22 \mathrm{~mm}$ x $22 \mathrm{~mm}$ cover slips (Thermo Fisher) in 6 well plates. $3 \times 10^{5}$ cells were seeded per well, incubated at $37^{\circ} \mathrm{C}$ and allowed to attach overnight. A 1:50 dilution (2\%) of sonicated MIPs (5 minutes with 30 seconds on-off intervals, using a Diagenode Bioruptor 2000 standard sonicator) was added to the media and incubated at $37^{\circ} \mathrm{C}$ for 24 hours. The following day, cells fixed with formalin, washed and then incubated with $1 \mu \mathrm{g} / \mathrm{ml}$ Diamidino-2-Phenylindole, Dihydrochloride (DAPI, Invitrogen) in the dark for 10 minutes. Anti-fade mounting medium was dropped on a microscope slide and the washed cover slip was gently laid on the slide. The edges were sealed with nail varnish. Slides were protected from light and imaged using the Leica TCS SP5 confocal laser scanning microscope (Leica Microsystems).

A modified antibody binding assay protocol was used to detect nanoMIPs binding to cells by flow cytometry. Cells were detached either by using trypsin or by scraping with ice cold PBS. Cells were counted, washed and re-suspended in ice cold FACS buffer (2\% FBS in $1 \mathrm{x}$ PBS) to obtain at least $1 \times 10^{6}$ cells/ml. Samples were transferred to round bottom 
polystyrene tubes (Falcon) on ice and fluorescent nanoMIPs were added to the cells and incubated in the dark for 10 minutes. Samples were immediately read on the BD FACS Canto II (Becton Dickenson Biosciences). Unstained controls were used to gate the positive populations. Acquisition and analysis of data was done using the FACS Diva ${ }^{\mathrm{TM}}$ version 6.1.3 software (BD Biosciences). 